\title{
Papel da tuberculose domiciliar no surgimento da tuberculose multirresistente*
}

\author{
The role of household contact in the appearance of multidrug-resistant \\ tuberculosis \\ ELIZABETH CLARA BARROSO, ROSA MARIA SALANI MOTA, ADALGISA C. M. OLIVEIRA, JOSEFA \\ IVONETE R. CORDEIRO, JOANA BRASILEIRO BARROSO, JORGE LUIS NOBRE RODRIGUES
}

\begin{abstract}
Introdução: A tuberculose multirresistente é uma preocupação em todo o mundo. A identificação de fatores de risco associados pode contribuir para o seu controle.
\end{abstract}

Objetivo: Avaliar se o contato com tuberculose domiciliar é fator de risco para tuberculose multirresistente.

Métdodo: Estudo caso-controle de base populacional de modo retrospectivo. Foi considerado multirresistente o bacilo resistente a pelo menos rifampicina+isoniazida, e tuberculose sensível o caso no qual houvesse sido feito o primeiro tratamento num período semelhante ao primeiro tratamento do caso com tuberculose multirresistente, mas que estivesse curado no momento da entrevista. Os casos foram selecionados através dos testes de sensibilidade realizados no Laboratório Central do Estado do Ceará de 1990 a 1999, pelo método das proporções. Os controles foram selecionados entre os bacilíferos do livro de registro do Programa de Controle da Tuberculose, em período semelhante. Foi pesquisada a história de tuberculose na família. Os casos de Tuberculose na família foram divididos em 03 subgrupos: tuberculose curada, tuberculose abandonada e tuberculose multirresistente.

Resultados: 266 casos de tuberculose multirresistente foram diagnosticados. Identificamos 153 pacientes, dos quais, 19 foram excluídos. 0 grupo de casos e controle foi de 134 e 185 pacientes, respectivamente. Através do teste exato de Fisher não foi encontrada associação entre tuberculose multirresistente e contato com tuberculose na família $(p=0,1190)$. Estudando os subgrupos, encontramos que o contato com pacientes curados de tuberculose estava associado com tuberculose sensível $(p<0,0001)$, enquanto que o abandono do tratamento da tuberculose na família e a tuberculose multirresistente na família estavam associados com a tuberculose multirresistente, $p<0,0160$ e $p<0,0300$ respectivamente.

Conclusão: A existência na família de casos de tuberculose multirresistente e de casos de tuberculose com abandono de tratamento é fator de risco para tuberculose multirresistente e, portanto, constitui problema de saúde pública.

(J Bras Pneumol 2004; 30(1) 46-52

Descritores: Tuberculose/epidemiologia. Desistência do paciente. Fatores de risco.

Siglas e abreviaturas utilizadas neste trabalho

TB- Tuberculose

BK- Bacilo de Koch (SAI)

INH- Isoniazida

RFP- Rifampicina
Background: Multidrug-Resistant tuberculosis (MDR-TB) is a matter of worldwide concern. Identify associated risk factors may contribute to its control.

Objectives: To assess if household tuberculosis (TB) cases would is a risk factor for MDR-TB.

Method: A population-based case-control study was conducted in a retrospective way. Multidrug resistance was defined as resistance to at least Rifampin (RFM) and lsoniazid (INH), and susceptible TB (the case when first treatment had been made in a period similar to the first treatment of the MDR-TB cases, but disease free at the time of the interview). Selection of cases was made based upon the list of Susceptibility Tests (ST) carried out at the Central Laboratory of Public Health of the State of Ceará from, 1990 to 1999. The proportion method was used. Controls were selected from among the records of the TB Contro Program. The history of TB in the family was investigated. These cases were divided into three groups: cured TB, non adherence TB and MDR-TB.

Results: During the study period, 266 cases of MSR TB were diagnosed. We identified only 153 patients. Of these, 19 were excluded. The group of cases was comprised of 134 patients and that of controls by 185 . Fisher exact test disclosed no association between MDR-TB and household contacts with TB cases $(p=0.119)$. Studying the subgroups we found that contact with cured TB patients was associated with susceptible TB $(p<0.0001)$ whereas household non-adherence TB and in the family MDR-TB were associated with MDR-TB, $\mathrm{p}<0.016$ and $p<0.03$ respectively.

Conclusion: In the family cases of MDR-TB and cases of nonadherence to treatment are risk factors for MDR-TB and therefore represent a public health problem.

* Trabalho realizado como parte de Mestrado realizado sob orientação do Prof. Dr. Jorge Luis Nobre Rodrigues, cuja tese foi defendida em 09/10/01. Estudo realizado no Hospital de Maracanaú- Ministério da Saúde e Hospital de Messejana- Secretaria da Saúde do Estado do Ceará. Endereço para correspondência: Elizabeth Clara Barroso - Rua Fonseca Lobo 50 apto 402, Aldeota. CEP - 60.175-020 - Fortaleza, CE. Tel (85) 267-1557. E-mail - vbarroso@portalnet.com.br

Recebido para publicação, em 12/5/03. Aprovado, após revisão, em 10/09/03

Key words: Multidrug-resistant tuberculosis. Household contact. Non-adherence to tuberculosis treatment.

PZA- Pirazinamida

TBMR- Tuberculose multirresistente

TS- Teste de Sensibilidade

IC- Intervalo de confianç

Derease Control 


\section{INTRODUÇÃO}

Resistência às drogas é uma ameaça aos programas de controle da tuberculose (TB) em todo o mundo. Pacientes infectados com cepas resistentes a múltiplas drogas têm menor probabilidade de se curarem (1-5), particularmente se estão infectados pelo HIV ou sofrem de outra imunopatia. 0 tratamento é mais tóxico e 700 vezes mais caro que o tratamento de pacientes com organismos sensíveis ${ }^{(6-10)}$.

A tuberculose multirresistente (TBMR) é definida internacionalmente como o caso de portador de Mycobacterium tuberculosis resistente a pelo menos rifampicina (RFP) + isoniazida $(\mathrm{INH})^{(11)}$. A resistência primária do Mycobacterium tuberculosis à RFP, em serviço ambulatorial de referência em São Paulo, passou de 0,3\%, na década de 70, para $1,1 \%$, na década de $80(p=0,02)^{(3)}$. A prevalência combinada média mundial da TBMR encontrada pelo Projeto Global de Vigilância à Resistência às Drogas Antituberculose (1994-1997), foi de 5,0\%, no Brasil de $1,3 \%{ }^{(11)}$ e no Ceará de $1,1 \%{ }^{(12)}$. No Estado do Ceará houve um total de 41.073 casos de TB notificados, do ano de 1990 a $1999^{(12)}$, com uma tendência decrescente no decorrer do periodo. Em contraste, a incidência anual da TBMR apresentou tendência crescente, e a prevalência combinada de TBMR aumentou de 0,82\% em 1994 para 1,48\% em 1999 ${ }^{(12)}$.

Inúmeras pesquisas têm sido publicadas sobre fatores de risco para TBMR, pois o controle desses fatores de risco pode prevenir o desenvolvimento da TBMR. Os principais fatores de risco citados são: tratamentos irregulares, história de tratamentos anteriores, presença de cavidades pulmonares, tuberculose pulmonar extensa, alcoolismo, uso de drogas ilícitas, falta de escolaridade, baixa renda familiar, encarceramento, falta de moradia, e uso de abrigos públicos ${ }^{(1,9,13-17)}$. Encontram-se na literatura científica poucos estudos a respeito do contato com TB (com essa designação) e estes não monstram associação estatisticamente significante com a TBMR ${ }^{(18-20)}$.

No entanto, pesquisas de fatores de risco como encarceramento, permanência em abrigos públicos ou em clínicas para cuidados de enfermagem, têm o mesmo significado de pesquisa sobre TB domiciliar. Todas essas situações têm um ponto em comum: o risco de transmissão da TBMR. Em algumas dessas pesquisas, encontrou-se importante associação ${ }^{(13,14)}$.

A existência de um portador de TB sensível ou TBMR no convívio doméstico é motivo de preocupação para familiares que procuram os profissionais de saúde em busca de orientação. A TB domiciliar é de longa data reconhecida como fator de risco para novos casos de TB de modo geral ${ }^{(21)}$ e a conduta já é bem estabelecida nesses casos. 0 objetivo desse estudo foi esclarecer essa situação em relação à TBMR.

\section{MÉTODO}

Foi feito um estudo caso-controle aninhado a um estudo de coorte de base populacional.

O grupo dos casos foi constituido por pacientes portadores de TBMR. Adotou-se a definição internacional para TBMR, pela qual o bacilo deve ser resistente a pelo menos $\mathrm{RFP}+\mathrm{INH}$. 0 grupo controle foi constituído por portadores de tuberculose sensível, assim considerada como aquela em que o paciente tivesse feito o primeiro tratamento num período semelhante ao primeiro tratamento do caso com TBMR. 0 paciente deveria ter sido bacilífero no início do tratamento, ter se curado com o Esquema 1, ou seja, $2 \mathrm{RFP}+\mathrm{INH}+\mathrm{PZA}$ (pirazinamida) $/ 4 \mathrm{RFP}+\mathrm{INH}$, e estar curado no momento da entrevista.

Os casos foram selecionados tomando-se como base os 1500 testes de sensibilidade (TS) efetuados no Laboratório Central, único laboratório a fazer TS no Estado do Ceará e supervisionado pelo Centro de Referência Professor Hélio Fraga (Referência Nacional para realização de TS) no período de 1990 a 1999. O Ceará abriga uma população de 6.809.290 habitantes ${ }^{(22)}$ e sua capital, Fortaleza, 1.965.513 ${ }^{(22)}$.

Os serviços que contribuíram para a formação do grupo de casos foram o Hospital de Maracanaú que é referência estadual para internações em TB e se localiza em Maracanaú (CE), cidade que tem 
160.065 habitantes ${ }^{(22)}$, o Hospital de Messejana, localizado em Fortaleza, referência hospitalar e ambulatorial, em TB, para o Estado do Ceará e estados vizinhos, e a Unidade Sanitária Dona Libânia, referência para atendimento ambulatorial, também localizada em Fortaleza.

Os controles foram selecionados de modo que houvesse um pareamento por gênero, idade e ano do primeiro tratamento. Foi calculado o período entre o primeiro tratamento e o diagnóstico de TBMR dos casos. Encontrou-se uma média de $6,5 \pm 3$ anos ( \pm desvio padrão). A pesquisa foi feita no ano 2000. Considerou-se o tempo necessário para o desenvolvimento de resistência e recuou-se a média mais um ano, começando-se a selecionar todos os casos bacilíferos do livro de registro de tratamento da TB (Hospitais de Maracanaú e de Messejana) a partir de 1993. Para as diversas faixas etárias ${ }^{(10-19,20-24)}$ e gênero selecionou-se o quádruplo dos casos, porque se sabia que os endereços de muitos poderiam ter mudado, que alguns poderiam ter falecido e que outros não atenderiam ao chamado para avaliação clínica e entrevista. Ao findar essa fase de seleção, não foi necessário fazer sorteio porque a quantidade selecionada já era a pré-estipulada. Foram enviadas correspondências, as quais tornavam os pacientes cientes do estudo. Ao comparecerem ao serviço de tisiologia, eles assinaram o termo de consentimento aprovado pelo Comitê de Ética em Pesquisa da Universidade Federal do Ceará, submeteram-se ao radiografia simples de tórax, a exames rotineiros de sangue (incluindo sorologia para HIV, se concordassem) e à pesquisa de BAAR no escarro (se tivessem expectoração). Se 0 paciente estivesse curado, preenchia-se o questionário que alimentaria o banco de dados dos controles. Os questionários dos casos e controles foram preenchidos pelo autor principal da pesquisa.

As definições do questionário são as descritas a seguir. Foi considerado TB domiciliar qualquer caso em que houvesse co-habitação de TB. Os casos de TB no domicílio foram estratificados em cura, abandono e TBMR, assim definidos: cura: o familiar com TB havia obtido cura e permanecia curado, segundo informação do caso ou controle (a maioria desses contatos havia sido tratada pelos autores); abandono: o familiar com TB havia abandonado o tratamento, segundo informação do caso ou controle; e TBMR: familiar com TBMR comprovada por TS (todos já conhecidos dos autores). Cura e abandono de tratamento foram definidos de acordo com $01^{\circ}$ Consenso Brasileiro de Tuberculose ${ }^{(23)}$. Casos com sorologia (+) para o HIV foram considerados $\mathrm{HIV}+$.

Foi utilizado o meio de cultura de Löwenstein Jensen. Para o TS utilizou-se o método das proporções com meio sólido e a resistência foi definida como crescimento de pelo menos $1 \%$ de colônias sob concentrações críticas das seguintes drogas: $0,2 \mu \mathrm{g} / \mathrm{mL}$ para isoniazida, $2 \mu \mathrm{g} / \mathrm{mL}$ para etambutol e $40 \mu \mathrm{g} / \mathrm{mL}$ para rifampicina; e crescimento de pelo menos 10\% de colônias sob concentrações críticas das seguintes drogas: $20 \mu \mathrm{g} / \mathrm{mL}$ para etionamida, 100 $\mu \mathrm{g} / \mathrm{mL}$ para pirazinamida e $4 \mu \mathrm{g} / \mathrm{mL}$ para estreptomicina.

A análise estatística para a comparação entre os grupos (caso e controle) foi realizada através do teste exato de Fisher para as variáveis independentes dicotomizadas e pela regressão logística para as variáveis independentes contínuas. Estimativas do Odds Ratio e intervalo de confiança (IC) com um coeficiente de confiança igual a 95\% também foram calculados. Foi considerado significativo um valor de $p<0,05$. As análises estatísticas referidas foram realizadas com o auxílio dos programas de computação SPSS versão 10.0.

\section{RESULTADOS}

Houve um total de 41.073 casos de TB notificados no Ceará, no período de 1990 a $1999^{122}$. As notificações da TB mostraram tendência decrescente no decorrer do periodo enquanto que a incidência anual da TBMR apresentou tendência crescente ${ }^{(12)}$. 0 estado abriga uma população de 6.809.290 
habitantes e sua capital, Fortaleza, de 1.965.513 habitantes ${ }^{(23)}$ e é responsável por 50\% das notificações de TB do estado. A Unidade Sanitária Dona Libânia e o Hospital de Messejana, dois serviços estaduais de referência ambulatorial para atendimento de $\mathrm{TB}$, contribuíram com pequena parte dos pacientes para a pesquisa. O Hospital de Maracanaú, que contribuiu com a quase totalidade dos pacientes do estudo, era referência estadual para internação em TB até a época da pesquisa. Localiza-se na cidade de Maracanaú, que tem 160.065 habitantes ${ }^{(23)}$ e é vizinha a Fortaleza.

Dos 1.500 TSs realizados no Laboratório Central do Ceará, na década de 90, 266 cepas eram resistentes a pelo menos RFP+INH. Foram identificados 153 desses pacientes, para os quais foi preenchido o questionário padrão. Destes, cinco foram excluidos por se tratarem de portadores de micobactérias atípicas e dois por não corresponderem à definição de TBMR adotada. Dos 146 casos, 12 não tinham história de tratamento anterior (TBMR primária) e foram excluídos, pois este era originalmente um estudo para TBMR adquirida. 0 grupo dos casos ficou composto por 134 pacientes.

Para a seleção dos controles, foram revistos 615 prontuários e enviadas correspondências para 504 pacientes. Os outros 111 foram excluídos por se tratarem de óbitos, transferências, etc. Das 504 cartas enviadas, 114 foram devolvidas pelo correio, e apenas 188 atenderam ao chamado. Dos 188, um paciente tinha diagnóstico de TBMR e passou para o grupo dos casos, e dois encontravam-se sintomáticos e foram excluídos da pesquisa, porque apesar de estarem abacilíferos, não havia tempo para esperar o resultado da cultura para bacilo de Koch e TS. Assim, o grupo controle ficou constituído de 185 pacientes.

Em relação ao período entre 0 primeiro tratamento e o diagnóstico de TBMR para os casos, encontramos uma média de $6,5 \pm 3$ anos ( \pm desvio padrão), e entre o primeiro tratamento e a data da entrevista, para os controles uma média de $6,5 \pm 4$ anos, o que mostrou que o pareamento buscado foi alcançado. A distribuição por gênero e idade dos pacientes e a associação encontrada com a TBMR estão expressas na Tabela 1 , onde se pode constatar o pareamento pretendido. Não houve casos HIV+ nos grupos.

A distribuição dos pacientes por grupo em função da TB no domicílio e em função de cada um dos três subgrupos estudados (TB curada na família, TB abandonada na família e TBMR na família) encontra-se na Tabela 2 .

Estimando através do $0 d d s$ ratio (OR), a chance no grupo dos pacientes com TB abandonada na família terem TBMR quando comparado com o grupo de pacientes sem TB abandonada, temos: $O R=3,63$; IC 95\% [1,29-10,19]. Portanto, a chance de um paciente ter TBMR no grupo em que existe TB abandonada na família é 3,63 vezes a chance de um paciente ter TBMR no grupo em que não existe TB abandonada na família. A chance entre os que têm TBMR na família de desenvolverem TBMR quando comparados com os que não têm TBMR na família é 3,83, com 1C 95\% [1,15-12,76]. Logo, a chance de um paciente ter TBMR no grupo em que existe TBMR na família é 3,83 vezes a chance de um paciente ter TBMR no grupo em que não existe TBMR na família (Tabela 3).

TABELA 1

Distribuição por gênero e idade dos 319 pacientes com tuberculose pulmonar

\begin{tabular}{lccc}
\hline Características & Casos $(\mathrm{n}=134)$ & Controles $(\mathrm{n}=185)$ & Valor de $\mathrm{p}$ \\
\hline Sexo masculino $-\mathrm{n}^{\circ}(\%)$ & $81(60,40)$ & $116(62,70)$ & $0,7270^{*}$ \\
Média de idade - anos $\pm \mathrm{DP}$ & $39,78 \pm 13,26$ & $41,22 \pm 14,14$ & $0,3591+$
\end{tabular}

$\mathrm{DP}=$ desvio padrão; * teste exato de Fisher; ${ }^{\dagger}$ regressão logistica. 
TABELA 2

Distribuição dos pacientes com tuberculose pulmonar, por grupo, em função da tuberculose(TB) no domicílio e de cada um dos 03 subgrupos: TB curada no domicílio, TB abandonada no domicílio e TBMR no domicílio

\begin{tabular}{lllll}
\hline & & Controles & Casos & Valor de p* $^{*}$ \\
\hline \multirow{2}{*}{ TB no domicílio } & & $\mathrm{N}(\%)$ & $\mathrm{N}(\%)$ & \\
& Não & $128(69,2)$ & $79(60,3)$ & $\mathrm{P}=0,1190$ \\
& Sim & $57(30,8)$ & $52(39,7)$ & \\
& Total & $185(100,0)$ & $131(100,0)$ & \\
TB curada no domicílio & Não & $3(5,3)$ & $23(44,2)$ & $\mathrm{P}<0,0001$ \\
& Sim & $54(94,7)$ & $29(55,8)$ & \\
& Total & $57(100,0)$ & $52(100,0)$ & \\
TB abandonada no domicílio & Não & $51(89,5)$ & $36(69,2)$ & $\mathrm{P}=0,0160$ \\
& Sim & $6(10,5)$ & $16(30,8)$ & \\
& Total & $57(100,0)$ & $52(100,0)$ & \\
TBMR no domicílio & Não & $53(93,0)$ & $40(76,9)$ & $\mathrm{P}=0,0300$ \\
& Sim & $4(7,0)$ & $12(23,1)$ & \\
& Total & $57(100,0)$ & $52(100,0)$ &
\end{tabular}

* Teste exato de Fisher.

\section{DISCUSSÃO}

Os autores, inicialmente, chamam a atenção para as limitações próprias dos estudos retrospectivos, em que viés pode resultar da revisão dos prontuários, da confiabilidade dos dados neles existentes e até do preenchimento dos questionários, mesmo na presença dos pacientes, por lapsos de memória. Neste estudo, os pacientes tiveram de rememorar fatos ocorridos há aproximadamente $6,5 \pm 3$ anos ( \pm desvio padrão). No grupo dos casos, apenas $25 \%$ dos pacientes tiveram seus dados colhidos por prontuário, sem a presença deles, mas como se tratavam de casos crônicos, eles tinham numerosas internações e os prontuários, muitas informações. Para evitar viés por falta de dados ou falta de credibilidade, os autores recorreram ainda às profissionais de enfermagem e do serviço social, as quais, além de terem sido responsáveis por boa parte das informações de prontuário,

TABELA 3

Estimativa, através do Odds Ratio (OR), da chance de desenvolvimento de TBMR em função da tuberculose abandonada ou TBMR no domicílio

\begin{tabular}{lll}
\hline & OR & IC (95\%) \\
\hline TB abandonada no domicílio & 3,63 & {$[1,29-10,19]$} \\
TBMR no domicílio & 3,83 & {$[1,15-12,76]$} \\
\hline
\end{tabular}

TB: tuberculose; TBMR: tuberculose multirresistente; IC: intervalo de confiança. conheciam os pacientes muito bem, devido às numerosas consultas e internações. Os outros 75\% vinham sendo acompanhados pelos autores do estudo na última década e estavam presentes ao preenchimento do questionário. Quanto ao grupo dos controles, os dados foram colhidos pelo autor na presença do paciente e com a utilização concomitante do prontuário, o que permitiu confrontar-se os dados.

Foi feito pareamento por gênero e faixa etária porque se achou que essas variáveis poderiam funcionar como fatores confundidores, já que na literatura científica encontram-se resultados bastante discrepantes em relação a essas duas características ${ }^{(15-17)}$.

Boudville et al. publicaram em 1997 um estudo caso-controle, realizado em Cingapura, para pesquisa de fatores de risco para TBMR, no qual, 10 entre 134 (7\%) casos tinham história de contato com TB (9 domiciliares e 1 no trabalho) e 17 entre 207 $(8 \%)$ controles também a tinham, não tendo sido encontrada significância estatística ${ }^{(19)}$.

Estudo caso-controle, para pesquisa de fatores de risco para resistência a quimioterapia antituberculose, realizado por Natal et al., nos 23 centros de saúde do Estado Rio de Janeiro e publicado em 2000, também, não demonstrou associação entre TBMR e história de contato com TB ${ }^{(20)}$.

No presente estudo encontrou-se um percentual de 30,8\% $(57 / 128)$ de ТВ no domicílio entre os controles e 39,7\% (52/131) entre os casos. Esses achados são semelhantes do ponto de vista estatístico $(P=0,1190)$.

Em estudo transversal multihospitalar, realizado em Madrid, para estudar a resistência a fármacos antituberculose, publicado em 1996, Peña et al. incluíram contato com TB entre as características epidemiológicas dos pacientes, mas apresentaram dados apenas descritivos onde de 467 pacientes, 14\% tinha história de contato com $\mathrm{TB}^{(18)}$.

Estudando uma série de 70 portadores de cepas multirresistentes, em 1993 e 1994, no Instituto Clemente Ferreira de São Paulo, 
Seiscento et al. encontraram 8,6\% (6/70) com história de contato com TBMR ${ }^{(4)}$.

Melo et al. conduziram estudo de uma coorte de pacientes diagnosticados como portadores de TBMR pulmonar, de 1995 a 1999, no mesmo serviço acima. Dos 170 pacientes que forneceram informações, 129 tinham contágio desconhecido e 41 (29\%) identificados, sendo 34/41 (83\%) intradomiciliares e 7/41 (17\%) institucionais. Dos 34 com contacto intradomiciliar, 15 foram considerados contactantes do caso índice ou de foco não considerado multirresistente, e 19 com foco de TBMR. No presente estudo, houve 29 casos de TB curada no grupo com TBMR, contra 12 de TBMR persistente. À primeira vista, parece haver associação da TB curada com a TBMR. Ao se fazer a análise comparativa com o grupo controle, comprova-se a associação da TBMR com a existência de TBMR no domicílio ${ }^{(24)}$.

Foram revisadas outras pesquisas de fatores de risco como encarceramento, permanência em abrigos públicos ou em clínicas para cuidados de enfermagem, que têm o mesmo significado da pesquisa a respeito de $\mathrm{TB}$ na família, pois todas essas situações têm em comum o risco de transmissão da TBMR. Em algumas dessas pesquisas encontrou-se associação significante ${ }^{(13,14)}$.

A divisão do grupo TB no domicílio nos subgrupos TB curada, TB abandonada e TBMR foi fundamental para se entender porque o contato com TB, de modo geral, não é fator de risco para TBMR. Observando-se a Tabela 2, pode-se ver que o percentual de TB no domicílio entre casos e controles é semelhante, mas ao se fazer a estratificação, a TB curada no domicílio se associa de modo importante $(p<0,0001)$ à TB sensivel (controles) enquanto a TB abandonada na família e a TBMR na família associam-se com a TBMR $(p<0,0160$ e $p<0,0300$ respectivamente). Estimando-se através do $\mathrm{OR}$, a chance de desenvolvimento de TBMR em famílias com TB abandonada ou TBMR é cerca de quatro vezes maior que a chance em famílias sem esses fatores de risco.

Assim é possivel concluir que a TB tratada corretamente e curada na família não oferece risco para TBMR. A existência de TB abandonada e TBMR na família são fatores de risco para TBMR e, portanto, constituem problemas de saúde pública. Medidas de proteção e orientação para familiares de portadores de TB abandonada e TBMR precisam ser estabelecidas. Medidas para o controle do abandono precisam ser incentivadas. Entre outras medidas proporcionadas pelo serviço de saúde, poderia se convencer a família da grande importância do seu apoio ao paciente, já que o abandono constitui risco também para o restante da família. Inclusive a internação compulsória seria uma alternativa. Finalmente, um esquema de quimioprofilaxia adequado precisa ser instituido para os contatos domiciliares de TBMR.

\section{Referências:}

1. Goble M, Iseman MD, Madsen LA, Waite D, Ackerson 1 , Horsburgh Jr CR. Treatment of 171 patients with pulmonary tuberculosis resistant to isoniazid and rifanpin. N Engl $J$ Med 1993;328:527-32

2. Fiúza de Melo FA, Ide Netto J, Seiscento M, Pinto JA, Afiune JB. Tuberculose multirresistente. J Pneumol 1993;19:73-81.

3. Fiúza de Melo FA, Afiune JB, Ribeiro LHG, Felice EAA, Castelo A. Resistência primária do $M$. tuberculosis num serviço ambulatorial de referência em São Paulo: evolução por três décadas e comparação com outros estudos nacionais. J Pneumol 1996;22:3-8.

4. Seiscento M, Melo FAF, lde Neto J, Noronha AML, Afiune JB, Inomat T, Cruz ML. Tuberculose multirresistente (TBMR): aspectos clínico-laboratoriais, epidemiológicos e terapêuticos. J Pneumol 1997;23:237-44.

5. Dalcolmo MP, Fortes A, Melo FAF, Motta R, lde Neto J, Cardoso N, Andrade M, Barreto AW, Gerhardt G. Estudo de efetividade de esquemas alternativos para tratamento da tuberculose multirresistente no Brasil. J Pneumo 1999;25:70-7.

6. Mahmoudi AMD, Michael D, Iseman MD. Pitfalls in the care of patients with tuberculosis: common errors and their association with the acquisition of drug resistance. JAMA 1993;270:65-8.

7. Humma LM. Prevention and treatment of drug-resistant tuberculosis. Am J Health Syst Pharm 1996;53:2291-8.

8. Heymann SJ, Brewer TF, Wilson ME, Fineberg HV. The need of global action against multidrug-resistant tuberculosis. JAMA 1999;281:2138-40.

9. Rao SN, Mookerjee AL, Obasanjo 00, Chaisson RE. Errors in the treatment of tuberculosis in Baltimore. Chest 2000;117:734-7. 

Science 2000;287:2391,

11. Pablos-Mendez A, Raviglione MC, Laszlo A, Binkin N, Rieder $\mathrm{H}$, Bustreo F, et al. Global surveillance for antituberculosisdrug resistance, 1994-1997. World Health Organization International Union against Tuberculosis and Lung Disease Working Group on Anti-tuberculosis Drug Resistance Surveillance. N Engl J Med;1998;338:1641-9.

12. Barroso EC, Rodrigues JLN, Pinheiro VGF, Campelo CL. Prevalência da tuberculose multirresistente no Estado do Ceará, 1990-1999. J Pneumol 2001;27:310-4.

13. Neville K. The third epidemic multidrug-resistant tuberculosis. Chest 1994;105:45-8.

14. Sandman L. Risk factors for rifampin-monoresistant tuberculosis: a case-control study. Am J Respir Crit Care Med 1999;152:468-72.

15. Garcia Rodriguez JF, Marino Callejo A, Lorenzo Garcia MV, Rodriguez Gomez D, Sesma Sanches P. Resistance of Mycobacterium tuberculosis in Ferrol, Spain. Associated factors. Med Clin (Barc) 1999;113:572-4.

16. Sifuentes-Osornio J, Ponce-de Leon LA, Camacho-Mesquita FE, Bobadilla-Del-Valle JM, Infante-Suarez ML, RamirezFernandez N, et al. Resistencia de Mycobacterium tuberculosis en pacientes mexicanos. 1. Caracteristicas clinicas y factores de riesgo. Rev Invest Clin 1995;47:273 81.

17. Arévalo M, Solera J, Cebrian D, Bartolome J, Robles P. Risk factors associated with drug resistant Mycobacterium tuberculosis in Castilla - La Mancha (Spain). Eur Respir 1996;9:274-8.

18. Peña JM, Ortega A. Estudio transversal multihospitalario de tuberculosis y resistencias en Madrid (octubre de 1993 abril de 1994). Med Clin (Barc) 1996;106:1-6.

19. Boudville IC, Wong SY, Snodgrass 1. Drug resistant tuberculosis in Singapore. Ann Acad Med Singapore 1997;26:549-56.

20. Natal S. Fatores de predição para a resistência aos tuberculostáticos [abstract]. Bol Pneumol Sanit 2000;8:645.

21. Rouillon A, Perdrizet S, Parrot R. Transmission of tubercle bacilli: the effects of chemotherapy. Tubercle 1976;57:27599.

22. Instituto Brasileiro de Geografia e Estatística. Ceará Contagem da população de 1996. [Acesso em 12 jun 2000]. Disponível

URL:http://ibge.gov.br/ibge/estatistica/populaçao/contagem /cecont96.shtm.

23. Coordenação Nacional de Pneumologia Sanitária. 1 Consenso Brasileiro de Tuberculose - 1997. J Pneumol 1997;23:294-319.

24. Melo FAF, Afiune JB, lde Neto J, Almeida EA, Spada DTA, Antelmo ANL, Cruz ML. Aspectos epidemiológicos da tuberculose multirresistente em serviço de referência na cidade de São Paulo. Rev Soc Bras Med Trop 2003;36:27-34 